\title{
Recent astrophysical studies with exotic beams at ORNL
}

\author{
D.W. Bardayan ${ }^{\mathrm{a}}$ \\ Physics Division, Oak Ridge National Laboratory, Oak Ridge, TN 37831, USA
}

Received: 17 June 2005 /

Published online: 28 February 2006 - C Società Italiana di Fisica / Springer-Verlag 2006

\begin{abstract}
The availability of exotic beams has produced great opportunities for advances in our understanding of the nucleosynthesis occurring in stellar burning and stellar explosions such as novae, X-ray bursts, and supernovae. In these extreme environments, synthesized radioactive nuclei can undergo subsequent nuclear processing before they decay, and thus to understand these events, we must understand reaction rates involving radioactive nuclei. At the ORNL Holifield Radioactive Ion Beam Facility (HRIBF), we have made a number of measurements using proton-rich beams such as ${ }^{18} \mathrm{~F}$ and ${ }^{7} \mathrm{Be}$ and neutron-rich beams such as ${ }^{82} \mathrm{Ge}$ and ${ }^{84} \mathrm{Se}$ that help clarify the structure of astrophysically-important nuclei. We are also poised to begin studies with doubly-magic ${ }^{132} \mathrm{Sn}$. The experimental methods and results are discussed.
\end{abstract}

PACS. 25.60.-t Reactions induced by unstable nuclei $-26.30 .+\mathrm{k}$ Nucleosynthesis in novae, supernovae and other explosive environments

\section{Introduction}

Nuclear astrophysics addresses some of the most compelling questions in nature: What are the origins of the elements that make life on earth possible? How did the sun, the solar system, the stars, and our galaxy form, and how did they evolve? What is the total density of matter in the universe, and will the universe eventually collapse or expand forever? Astrophysical models that address these crucial questions require a considerable amount of nuclear physics information as input. The majority of this required information, however, is currently based on extrapolations or theoretical models and does not have a firm experimental basis. Nuclear data is also an important ingredient in the interpretation of new observations made by ground-based observatories such as the Keck and European Southern Observatory (ESO) Very Large Telescopes, by space-borne observatories such as the Hubble Space Telescope and the Chandra X-Ray Observatory, and by large subterranean detectors such as the Sudbury Neutrino Observatory and Super-Kamiokande. More complete and precise nuclear physics measurements are therefore needed to improve astrophysical models and to decipher the latest observations [1].

Because radioactive nuclei play an influential, and in some cases the dominant, role in many cosmic phenomena, information on these nuclei is particularly important to improve our understanding of the processes that shape our universe. In the explosive environments

\footnotetext{
a Representing the RIBENS Collaboration; e-mail: bardayan@mail.phy . ornl.gov
}

of novae and X-ray bursts, hydrogen and helium react violently with heavier seed nuclei to produce protonrich nuclei via hot-CNO burning and the $\alpha p$ - and $r p$ processes. Neutron-induced nucleosynthesis may occur in the neutrino-wind-driven shock front of supernova explosions initiating the $r$-process and producing extremely neutron-rich nuclei. These cataclysmic stellar explosions produce reaction flows through nuclei far from the valley of beta stability. To understand and interpret observations of these events we must understand the nuclear reactions, the nuclear structure, and the decay mechanisms for unstable nuclei. Because the lifetimes of most of the nuclei of interest are too short for use as targets in experiments, the required information can best be obtained by producing energetic beams of radioactive ions.

\section{Experimental details}

At the ORNL Holifield Radioactive Ion Beam Facility (HRIBF), exotic beams are produced by the ion source on-line (ISOL) method [2]. Light ion beams, accelerated by the Oak Ridge Isochronous Cyclotron (ORIC), bombard thick, hot, refractory targets to produce radioactive atoms. The atoms diffusing from the target material are ionized and extracted by a close-coupled ion source. After undergoing two stages of mass analysis, the radioactive ions are then injected into the $25 \mathrm{MV}$ tandem accelerator, accelerated to the appropriate energy for the experiment, and delivered to the experimental station. At the HRIBF, a full suite of developed beams is available including 120 
radioactive beams and 79 beams of stable species [3]. Available radioactive beams include proton-rich species such as ${ }^{7} \mathrm{Be}$ and ${ }^{17,18} \mathrm{~F}$ and neutron-rich beams such as ${ }^{82} \mathrm{Ge}$ and doubly-magic ${ }^{132} \mathrm{Sn}$. Energies up to $\sim 10 \mathrm{MeV} / \mathrm{u}$ are possible for low mass beams and up to $\sim 5 \mathrm{MeV} / \mathrm{u}$ for beams near ${ }^{132} \mathrm{Sn}$.

While the availability of radioactive beams provides great opportunities for the better understanding of nuclei and nuclear reactions far from stability, there are also great challenges that must be overcome to utilize such beams. Radioactive beams are generally available only at a much lower intensity than stable beams ( $10^{4-7}$ ions/s compared to $10^{9-12}$ ions/s for stable beams). High-efficiency large solid-angle detector arrays have been developed to deal with low beam intensity measurements. The Silicon Detector Array (SIDAR) [4] is a segmented array of silicon strip detectors that has been used for several of the nuclear astrophysics measurements at the HRIBF. Other detector arrays available at the HRIBF include the CLARION array of Clover germanium detectors and the HYBALL array of CsI detectors [5]. Another challenge is that radioactive beams are frequently contaminated with an unwanted isobar. While this contaminant can sometimes be removed with chemical techniques at the ion source or by fully stripping the beam (usually with accompanying drops in intensity), a general solution does not exist and many times an isobarically mixed beam will be delivered to the experimenter. A detector system with high selectivity is many times the best solution to the problem. Kinematically-complete measurements utilizing coincidence techniques is one method to identify the events of interest in such cases. We have frequently detected the beam-like recoil from a reaction in a gas-filled ionization counter, identifying the proton number $(Z)$ of the recoil, and thus determining the reaction channel. Because the measurements of astrophysical interest are usually performed in inverse kinematics, the beam-like recoils are forward focused and can be efficiently detected in an ionization counter covering relatively small laboratory angles $\left(<10^{\circ}\right)$.

While direct measurements of the reaction of interest are extremely important, it is not generally possible (or even desirable) to measure the reaction cross-section at all of the energies required to completely determine the stellar reaction rate. It is crucial to have complimentary measurements which may not directly determine the reaction rate but do help to elucidate the structure of the compound nuclear system involved. A good understanding of the properties of states in the compound nuclei can be used to calculate the stellar reaction rate over a large range of astrophysical temperatures. Direct measurements of reaction cross-sections should thus be complimented with other studies such as single-nucleon transfer reactions populating states of interest or particle-decay branching ratio studies from stable beam measurements. These points are discussed further in the following sections describing our studies with ${ }^{18} \mathrm{~F}$ beams (sect. 3 ), ${ }^{7}$ Be beams (sect. 4), and neutron-rich beams (sect. 5).

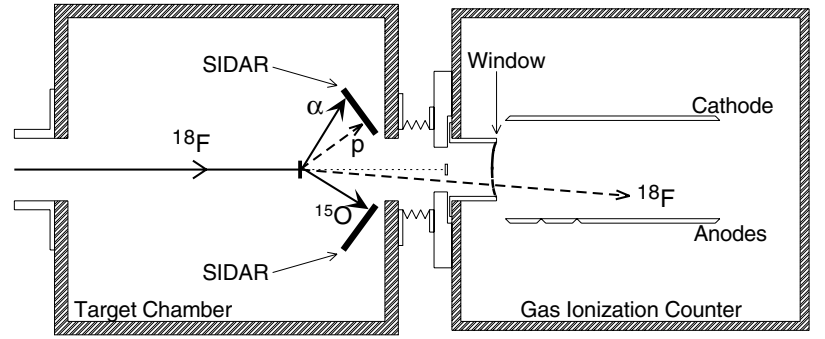

Fig. 1. Our experimental configuration is shown with the ${ }^{18} \mathrm{~F}$ ions impinging on a polypropylene target. For the ${ }^{1} \mathrm{H}\left({ }^{18} \mathrm{~F}, p\right){ }^{18} \mathrm{~F}$ measurement, scattered protons were detected in the SIDAR array in coincidence with recoil ${ }^{18} \mathrm{~F}$ ions detected by the ionization counter. For the ${ }^{1} \mathrm{H}\left({ }^{18} \mathrm{~F}, \alpha\right){ }^{15} \mathrm{O}$ measurement, both the recoil ${ }^{15} \mathrm{O}$ ions and $\alpha$ particles were detected in coincidence in the SIDAR.

\section{Studies with ${ }^{18} \mathrm{~F}$ beams}

Beams of ${ }^{18} \mathrm{~F}$ have been used to study ${ }^{18} \mathrm{~F}$ production in novae. ${ }^{18} \mathrm{~F}$ is relatively long-lived $\left(t_{1 / 2}=1.8 \mathrm{~h}\right)$ and produced in large amounts in novae. It is therefore a target for $\gamma$-ray astronomy, and its observation in novae would provide a rather direct test of nova models [6]. To interpret observations such as these, however, we must know the rates of thermonuclear reactions that affect ${ }^{18} \mathrm{~F}$ production. The ${ }^{18} \mathrm{~F}(p, \alpha){ }^{15} \mathrm{O}$ reaction is one of the most important reactions to understand as it destroys ${ }^{18} \mathrm{~F}$ in the nova environment but is also one of the most uncertain [7]. We have made several measurements at the HRIBF to better determine the ${ }^{18} \mathrm{~F}(p, \alpha){ }^{15} \mathrm{O}$ reaction rate in novae.

${ }^{18} \mathrm{~F}$ beams were produced by bombarding a fibrous $\mathrm{HfO}_{2}$ target with an $85 \mathrm{MeV}{ }^{4} \mathrm{He}$ beam. The ${ }^{16} \mathrm{O}(\alpha, p n){ }^{18} \mathrm{~F}$ reaction produced ${ }^{18} \mathrm{~F}$ atoms which were subsequently ionized, extracted, and accelerated by the HRIBF tandem electrostatic accelerator. Typical beam currents were $2 \times 10^{5}{ }^{18} \mathrm{~F} / \mathrm{s}$ with an ${ }^{18} \mathrm{O}$ contamination at a ratio of ${ }^{18} \mathrm{O} /{ }^{18} \mathrm{~F} \sim 8 / 1$. This ${ }^{18} \mathrm{O}$ contamination can be removed by stripping the beam to $q=9^{+}$before the energy-analyzing magnet with some loss in ${ }^{18} \mathrm{~F}$ beam intensity depending on the energy of the beam.

Our first measurement [8] was of the ${ }^{1} \mathrm{H}\left({ }^{18} \mathrm{~F}, p\right){ }^{18} \mathrm{~F}$ elastic-scattering cross-section as a function of energy. A ${ }^{18} \mathrm{~F}$ beam was used to bombard a hydrogen-containing $\left(\mathrm{CH}_{2}\right)_{\mathrm{n}}$ target and elastically-scattered protons were detected by the Silicon Detector Array (SIDAR) [4]. Beamlike recoils were detected and identified in coincidence at forward angles in an isobutane-filled gas ionization counter (see fig. 1). This coincidence requirement was necessary to discriminate protons scattered by ${ }^{18} \mathrm{~F}$ from protons scattered by ${ }^{18} \mathrm{O}$. The ${ }^{1} \mathrm{H}\left({ }^{18} \mathrm{~F}, p\right){ }^{18} \mathrm{~F}$ events were then counted as a function of beam energy to produce the excitation function shown in fig. 2. This study resolved significant uncertainties from previous discrepant measurements $[9,10]$ concerning the width and energy for an important $3 / 2^{+}$ resonance at $E_{\text {c.m. }}=665 \mathrm{keV}$.

This measurement has now been extended to a broader energy range $(0.3-1.3 \mathrm{MeV})$ [11] using the thick-target technique [12]. A $24 \mathrm{MeV}^{18} \mathrm{~F}$ was stopped in a thick 


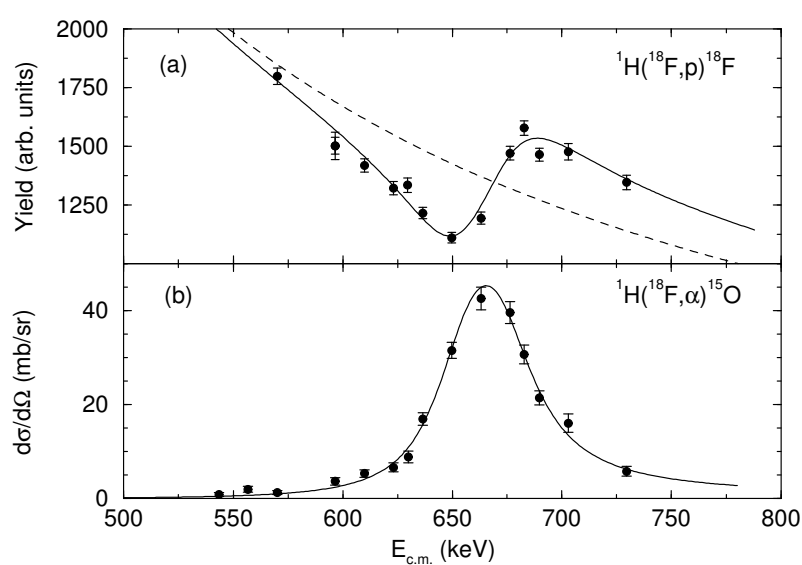

Fig. 2. (a) The ${ }^{1} \mathrm{H}\left({ }^{18} \mathrm{~F}, p\right){ }^{18} \mathrm{~F}$ excitation function is shown as a function of energy. The solid curve shows the best fit to the data, while the dashed curve shows the expected excitation function in the absence of resonances in this region. (b) The ${ }^{1} \mathrm{H}\left({ }^{18} \mathrm{~F}, \alpha\right){ }^{15} \mathrm{O}$ center of mass differential cross-section is plotted as a function of energy. The cross-section was found to be isotropic.

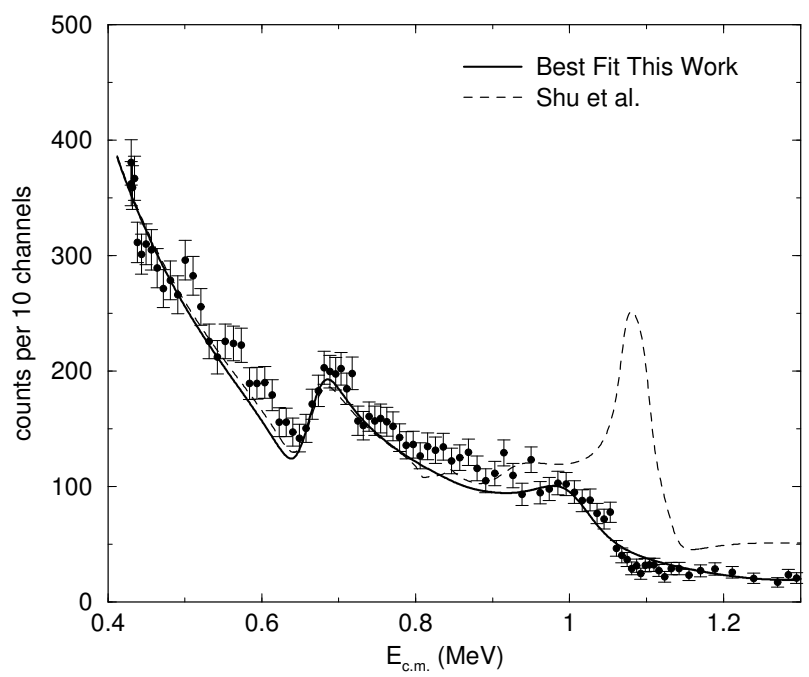

Fig. 3. The proton energy spectrum from the ${ }^{1} \mathrm{H}\left({ }^{18} \mathrm{~F}, p\right){ }^{18} \mathrm{~F}$ reaction at $\theta_{l a b}=12^{\circ}$ is shown. The solid line shows the best fit assuming a $\frac{7}{2}^{+}$resonance at $E_{c . m} \simeq 1.01 \mathrm{MeV}$. The dashed line shows the excitation function expected using the resonance parameters from ref. [13].

$2.8 \mathrm{mg} / \mathrm{cm}^{2}$ polypropylene target. The beam had been fully stripped to remove the ${ }^{18} \mathrm{O}$ contamination. Scattered protons from the ${ }^{1} \mathrm{H}\left({ }^{18} \mathrm{~F}, p\right){ }^{18} \mathrm{~F}$ reaction were detected at $\theta_{\text {lab }}=8^{\circ}-16^{\circ}$ by a double-sided silicon strip detector (DSSD). Because the scattered protons lose relatively little energy in the target, measurements of the proton's energy and angle of scatter were sufficient to determine the center-of-mass energy at which the reaction occurred [12]. A measurement of the scattered proton energy spectrum at a fixed angle can thus be used to extract the excitation function for the ${ }^{1} \mathrm{H}\left({ }^{18} \mathrm{~F}, p\right){ }^{18} \mathrm{~F}$ reaction over a wide range of center-of-mass energies. Using this technique, we measured the excitation function shown

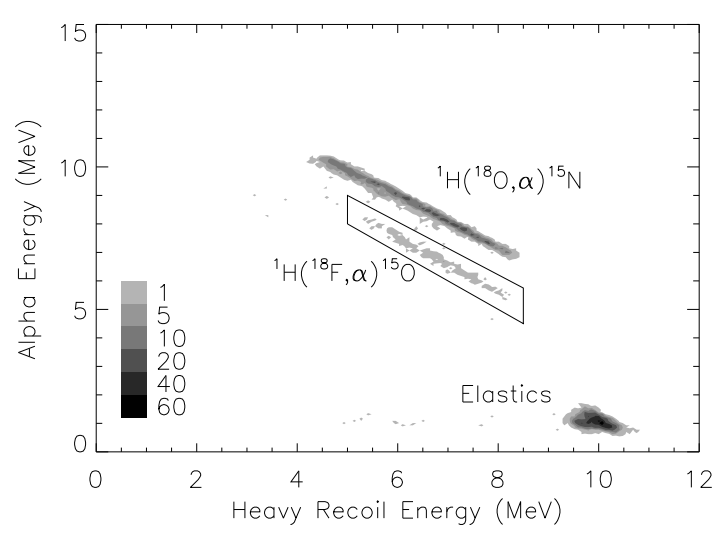

Fig. 4. The $\alpha$ particle energy is plotted vs. the heavy recoil energy. Owing to the different $Q$-values for the reactions, the ${ }^{1} \mathrm{H}\left({ }^{18} \mathrm{~F}, \alpha\right){ }^{15} \mathrm{O}$ events are readily distinguished from the ${ }^{1} \mathrm{H}\left({ }^{18} \mathrm{O}, \alpha\right){ }^{15} \mathrm{~N}$ events.

in fig. 3. The previously-observed resonance at $E_{c . m}$. $=$ $0.665 \mathrm{MeV}$ was clearly evident along with a new resonance at $E_{c . m} .=1.01 \mathrm{MeV}$. From an $R$-matrix fit to the data, we determined the most-likely properties of the latter to be $J^{\pi}=7 / 2^{+}, E_{x}=7.420 \pm 0.014 \mathrm{MeV}, \Gamma_{p}=27 \pm 4 \mathrm{keV}$, and $\Gamma_{\alpha}=71 \pm 11 \mathrm{keV}$. Possibly more important for novae temperatures, we were able to set upper limits on the proton widths of unobserved resonances, several of which are expected based upon comparisons with the mirror nucleus, ${ }^{19} \mathrm{~F}$. Because the proton widths are typically much smaller than the alpha widths for ${ }^{19} \mathrm{Ne}$ energy levels near the proton threshold $\left(E_{x}=6.411 \mathrm{MeV}\right)$, these upper limits directly constrain the resonance strengths and thus the contributions these unobserved levels could possibly make to the ${ }^{18} \mathrm{~F}(p, \alpha){ }^{15} \mathrm{O}$ rate.

In addition to the elastic-scattering measurements, we have also measured the ${ }^{1} \mathrm{H}\left({ }^{18} \mathrm{~F}, \alpha\right){ }^{15} \mathrm{O}$ cross-section directly for astrophysically-important resonances at $E_{c . m}$. 330 and $665 \mathrm{keV}$. The $665 \mathrm{keV}$ measurement was made simultaneously with the elastic-scattering measurement discussed above [14]. In this case, $\alpha$ particles and ${ }^{15} \mathrm{O}$ recoils were both detected in coincidence in the SIDAR array. The events of interest were identified by plotting (fig. 4) the detected $\alpha$ energy vs. the heavy recoil energy. Reactions for which both outgoing particles were detected appear as lines of constant total energy in fig. 4. Owing to the different $Q$ values for the reactions, the ${ }^{1} \mathrm{H}\left({ }^{18} \mathrm{~F}, \alpha\right){ }^{15} \mathrm{O}$ events were readily distinguished from the more intense ${ }^{1} \mathrm{H}\left({ }^{18} \mathrm{O}, \alpha\right){ }^{15} \mathrm{~N}$ events. By counting the number of ${ }^{1} \mathrm{H}\left({ }^{18} \mathrm{~F}, \alpha\right){ }^{15} \mathrm{O}$ events observed as a function of bombarding energy, the excitation function plotted in fig. 2(b) was produced. A simultaneous fit to the ${ }^{1} \mathrm{H}\left({ }^{18} \mathrm{~F}, p\right){ }^{18} \mathrm{~F}$ and ${ }^{1} \mathrm{H}\left({ }^{18} \mathrm{~F}, \alpha\right){ }^{15} \mathrm{O}$ data sets yielded a precise value of the resonance strength, $\omega \gamma=6.2 \pm 0.3 \mathrm{keV}$ [14]. This work also provided the first conclusive evidence for the $3 / 2^{+}$spin assignment of this resonance.

We have extended this technique to measure the resonance strength of an important $3 / 2^{-}$level at $E_{\text {c.m. }}$. $=$ $330 \mathrm{keV}$ [15]. Since the 1982 study by Wiescher and Ket- 


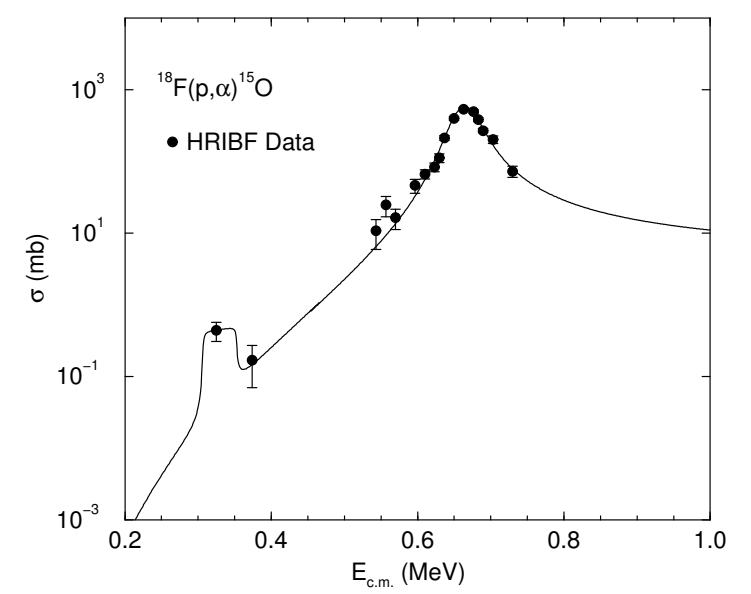

Fig. 5. The measured ${ }^{1} \mathrm{H}\left({ }^{18} \mathrm{~F}, \alpha\right){ }^{15} \mathrm{O}$ cross-section is shown along with a fit to the data. Since the width of the $330 \mathrm{keV}$ resonance is much less than the target energy loss, the curve appears "flat-topped" at these energies.

tner [16] of the ${ }^{18} \mathrm{~F}(p, \alpha){ }^{15} \mathrm{O}$ reaction, it has generally been believed that the reaction rate is dominated over a wide range of novae temperatures by a resonance at $E_{c . m .}=330 \pm 6 \mathrm{keV}$ that arises from a ${ }^{19} \mathrm{Ne}$ level at $E_{x}=6.741 \mathrm{MeV}$. This analysis and all subsequent calculations had to be based upon uncertain estimates of the strength of this resonance. Such estimates may be incorrect by an order of magnitude or more [7]. We have made the first significant measurement of the strength of this important resonance by measuring the yield of the ${ }^{1} \mathrm{H}\left({ }^{18} \mathrm{~F}, \alpha\right){ }^{15} \mathrm{O}$ reaction on and off resonance. Because the resonance energy was known well from previous studies and because the resonance is rather narrow $(\Gamma \sim 3 \mathrm{keV})$, we chose to measure the thick target yield by covering the energy range $\Delta E_{c . m} .=305-350 \mathrm{keV}$ within the target energy loss. In such a study, a measurement of the step height of the yield on resonance is directly related to the resonance strength of the state. The same technique as above was used to identify the events of interest, and the results are shown in fig. 5 along with the cross-sections from the $665 \mathrm{keV}$ study. The best fit to the data was obtained for a resonance strength of $\omega \gamma=1.48 \pm 0.46 \mathrm{eV}$ and a resonance energy of $332 \pm 17 \mathrm{keV}$ [15].

Additional constraints on the ${ }^{18} \mathrm{~F}(p, \alpha){ }^{15} \mathrm{O}$ reaction rate come from studies of the ${ }^{2} \mathrm{H}\left({ }^{18} \mathrm{~F}, p\right){ }^{19} \mathrm{~F}$ reaction. Levels in ${ }^{19} \mathrm{Ne}$ containing strong single-proton components should have mirror levels in ${ }^{19} \mathrm{~F}$ with strong single-neutron components. The isospin mirrors to astrophysically important ${ }^{19} \mathrm{Ne}$ levels should thus be strongly populated in a study of the ${ }^{2} \mathrm{H}\left({ }^{18} \mathrm{~F}, p\right){ }^{19} \mathrm{~F}$ reaction. Furthermore from the observed magnitudes of the cross-sections to populate the various states, one can use isospin-symmetry arguments to constrain the proton-spectroscopic factors (and thus the proton widths) of important ${ }^{19} \mathrm{Ne}$ levels.

The ${ }^{2} \mathrm{H}\left({ }^{18} \mathrm{~F}, p\right){ }^{19} \mathrm{~F}$ reaction was studied at the HRIBF [17] by bombarding a $160(10) \mu \mathrm{g} / \mathrm{cm}^{2}\left(\mathrm{CD}_{2}\right)_{n}$ target of $98 \%$ enrichment for $\sim 3$ days with an isotopically pure, $108.49 \mathrm{MeV}{ }^{18} \mathrm{~F}^{+9}$ beam at an intensity of $\sim 5 \times 10^{5} / \mathrm{s}$. Using the SIDAR array of $\sim 500 \mu \mathrm{m}$ thickness,

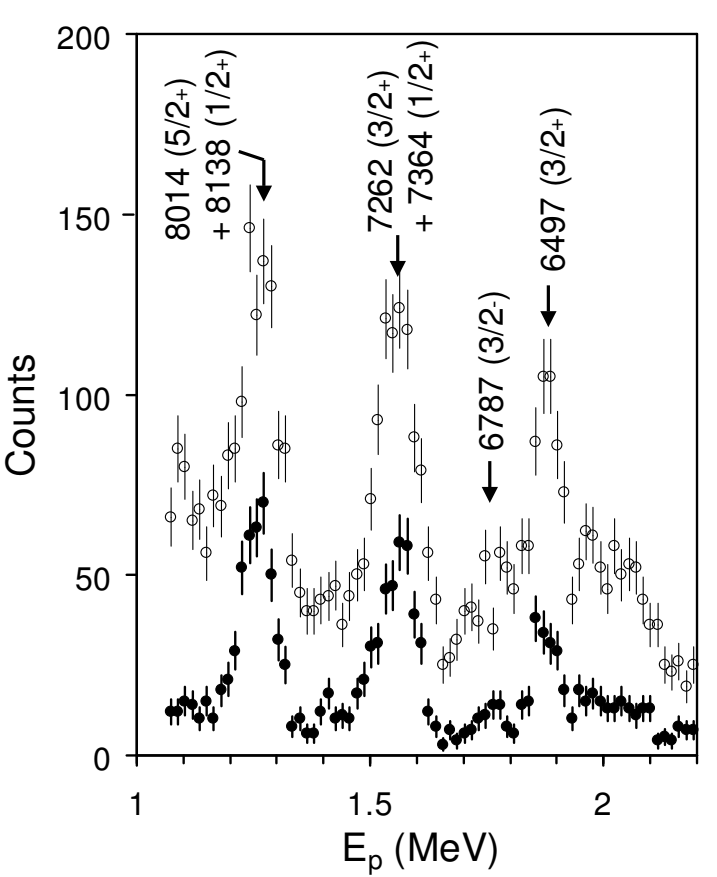

Fig. 6. ${ }^{2} \mathrm{H}\left({ }^{18} \mathrm{~F}, p\right){ }^{19} \mathrm{~F}$ spectra from the six SIDAR strips corresponding to a $147^{\circ}$ laboratory angle as a function of proton energy, displayed in $15 \mathrm{keV}$ bins. Open points are singles data and solid points are events in coincidence with the annular strip detector. Excitation energies are given in $\mathrm{keV}$.

light charged particles were detected in the laboratory angular range of $118^{\circ}-157^{\circ}$, corresponding to "forward" center-of-mass angles in the range $8^{\circ}-27^{\circ}$. The beam energy was selected to be high enough for direct reaction models, yet low enough to allow all the protons to be stopped in the SIDAR. A silicon strip detector at the focal plane of the Daresbury Recoil Separator (DRS) [18] was used to detect particle-stable recoils having $A=19$ in coincidence with the SIDAR. This coincidence efficiency was essentially $100 \%$ of the +9 charge state fraction for recoil angles $<1.6^{\circ}$ and $>70 \%$ overall for particle-stable final states. Other recoils, from higher, $\alpha$-decaying states in ${ }^{19} \mathrm{~F}$, were detected in coincidence just downstream from the target with an annular strip detector. This detector was also used for data normalization. Beam current normalization was achieved by directly counting beam particles at low intensity with a retractable silicon surface barrier detector placed temporarily at $0^{\circ}$. The overall uncertainty in normalization, estimated to be $\sim 10 \%$, is owing mostly to uncertainty in target thickness. Independent internal energy calibrations were obtained for each laboratory angle by using excitation energies of the well-known levels at 1.554038(9), 4.377700(42), and 5.1066(9) MeV in ${ }^{19} \mathrm{~F}$. This allowed excitation energies in the region of interest to be determined with uncertainties $\sim 10 \mathrm{keV}$.

Singles and coincidence spectra in the ( $\alpha$-decaying) region of importance for novae are shown for a laboratory angle of $147^{\circ}$ in fig. 6 . The coincidence spectra contain three main groups at about $6.5,7.3$, and $8.1 \mathrm{MeV}$ excitation energy. The coincidence efficiencies are roughly $50 \%$, 


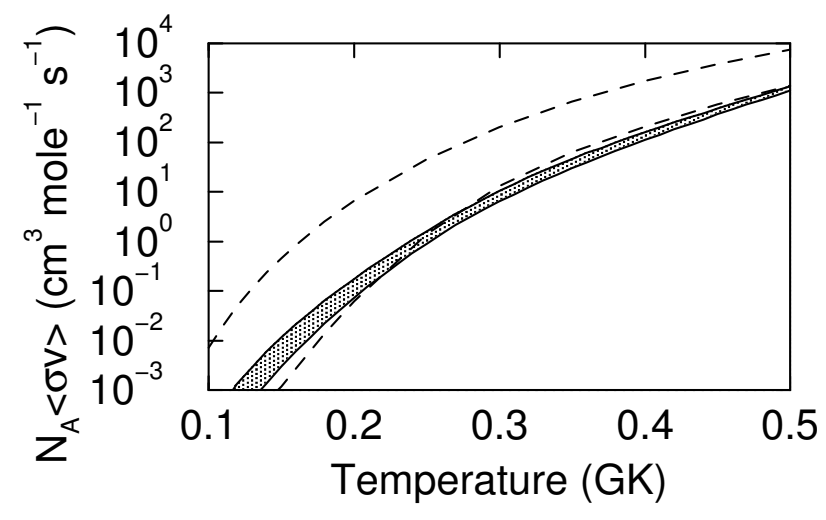

Fig. 7. The ${ }^{18} \mathrm{~F}(p, \alpha){ }^{15} \mathrm{O}$ reaction rate is plotted vs. temperature. The shaded band is from this work while the dashed band is from ref. [7].

$60 \%$, and $70 \%$, respectively. Our internal energy calibrations allow identifications of these states which are consistent with known levels in ${ }^{19} \mathrm{~F}$, as indicated in fig. 6 . Considerable strength was observed in the $6497 \mathrm{keV} 3 / 2^{+}$ ${ }^{19} \mathrm{~F}$ level, while we see no evidence for the $6528 \mathrm{keV}$ state as suggested in ref. [19]. This indicates that the $\ell=0$ strength near the proton threshold in ${ }^{19} \mathrm{Ne}$ may well be concentrated in a proton-bound state, or perhaps in the $8 \mathrm{keV}$ resonance. Furthermore, no evidence was observed for a strong $3 / 2^{+}$resonance reported previously at $7101 \mathrm{keV}$ [20]. Most likely the mirror to the important ${ }^{19} \mathrm{Ne}$ resonance at $665 \mathrm{keV}$ is thus contained in the doublet observed near $\sim 7.3 \mathrm{MeV}$ in fig. 6 . This is consistent with the results of recent reanalysis of ${ }^{15} \mathrm{~N}(\alpha, \alpha){ }^{15} \mathrm{~N}$ data [21].

Combining these various pieces of information on ${ }^{19} \mathrm{Ne}$ resonances, we have calculated improved values of the ${ }^{18} \mathrm{~F}(p, \alpha){ }^{15} \mathrm{O}$ reaction rate as a function of temperature [17]. In fig. 7, we plot our calculated rate as the shaded band along with the rate from ref. [7]. We find the rate to be dominated by resonances at $E_{c . m} .=330$ and $665 \mathrm{keV}$ for temperatures above $0.15 \mathrm{GK}$ and by the resonance at $38 \mathrm{keV}$ for temperatures below this. The rate is about two to five times smaller than that of Coc et al. [7] in the nova temperature range. We have used this updated ${ }^{18} \mathrm{~F}(p, \alpha){ }^{15} \mathrm{O}$ reaction rate in a nova nucleosynthesis calculation based on a post-processing approach with temperature and density histories of 28 zones of ejected material determined from hydrodynamics calculations of an explosion on the surface of a 1.25 solar mass white dwarf star [22]. The tools for this calculation are online in the Computational Infrastructure for Nuclear Astrophysics [23]. Using these new rates, we find roughly a factor of two more ${ }^{18} \mathrm{~F}$ is produced than previously calculated, and thus ${ }^{18} \mathrm{~F}$ observations are more likely than previously thought.

\section{Studies with ${ }^{7} \mathrm{Be}$ beams}

Interpretation of solar neutrino flux measurements requires an accurate knowledge of the ${ }^{7} \mathrm{Be}(p, \gamma)^{8} \mathrm{~B}$ cross-

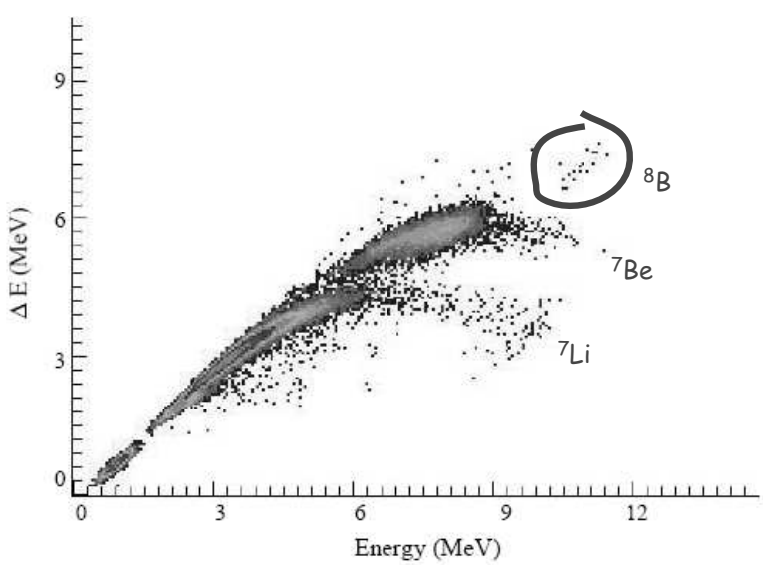

Fig. 8. ${ }^{8} \mathrm{~B}$ recoils from the ${ }^{7} \mathrm{Be}(p, \gamma){ }^{8} \mathrm{~B}$ reaction are identified in an ionization counter at the focal plane of the DRS. Scattered low-energy projectiles of ${ }^{7} \mathrm{Li}$ and ${ }^{7} \mathrm{Be}$ are also observed. $22{ }^{8} \mathrm{~B}$ events were identified in this demonstration experiment.

section. There have been over a dozen measurements of the reaction cross-section at a variety of energies, but all relatively precise measurements have used a radioactive (i.e., decaying) ${ }^{7} \mathrm{Be}$ target and thus have similar systematic uncertainties. To achieve the desired precision, different approaches, which are not inhibited with the same systematic effects, are necessary.

The present work [24] focuses on the development of a new approach for the measurement of ${ }^{7} \mathrm{Be}(p, \gamma)^{8} \mathrm{~B}$, using a ${ }^{7} \mathrm{Be}$ beam and a windowless hydrogen gas target at ORNL. The possible systematic errors for this measurement are different from those in normal kinematics using ${ }^{7}$ Be targets. In this case, the target is known to be pure $\mathrm{H}_{2}$ and to be of intransigent composition and thickness. Furthermore, the transmission target allows the recoils to be detected directly, and the systematic error in the number of fusions is decreased in the ${ }^{1} \mathrm{H}\left({ }^{7} \mathrm{Be}, \gamma\right){ }^{8} \mathrm{~B}$ measurement, due to high recoil-detection efficiency and low background. However, low ${ }^{7}$ Be beam current limits the statistical precision of the measurement. Due to the statistical limitations, our initial efforts will focus on the higher energy region of the cross-sections $\left(E_{c . m .}=1-2 \mathrm{MeV}\right)$, where the cross-section is highest and where the largest discrepancy exists among the various models used to extrapolate the cross-section to stellar energies [25].

The ${ }^{7} \mathrm{Be}$ used to make the radioactive beam was produced at the Triangle Universities Nuclear Laboratory (TUNL) using the ${ }^{7} \mathrm{Li}(p, n)^{7}$ Be reaction. A chemical process was performed to purify the ${ }^{7} \mathrm{BeO}$ material, which was mixed with copper or silver in a powder matrix and pressed into a pellet for use in a sputter ion source at the HRIBF. The sputter source produced a low energy beam of ${ }^{7} \mathrm{BeO}^{-}$which was injected into the tandem accelerator where it was broken up at the terminal by a gas stripper, and ${ }^{7} \mathrm{Be}^{1+}$ was mass analyzed and accelerated to the experiment.

Using this technique, a $12 \mathrm{MeV}{ }^{7} \mathrm{Be}$ beam was delivered to the HRIBF windowless hydrogen gas target [18] at an intensity of $2.5 \mathrm{ppA}$ and mixed with ${ }^{7} \mathrm{Li}$ at a ratio of 
${ }^{7} \mathrm{Be} /{ }^{7} \mathrm{Li} \sim 1 / 7$. The windowless gas target is described in detail in ref. [18]. The ${ }^{8} \mathrm{~B}$ recoils were tuned through the DRS, and counted and identified in the gas-filled ionization counter. The spectrum from the ion counter is shown in fig. 8. In this initial test measurement, $22{ }^{8} \mathrm{~B}$ recoils were observed resulting in a rough cross-section measurement of $1.12 \mu \mathrm{b}$ with $24 \%$ uncertainty at $E_{c . m} .=1.5 \mathrm{MeV}$. Further measurements with higher ${ }^{7} \mathrm{Be}$ beam intensities are planned to obtain a more statistically significant value.

Another stellar burning reaction studied at the HRIBF with ${ }^{7} \mathrm{Be}$ beams was the ${ }^{3} \mathrm{He}\left({ }^{3} \mathrm{He}, 2 p\right){ }^{4} \mathrm{He}$ reaction. Approximately $86 \%$ of the hydrogen being processed to helium in the sun flows through the ${ }^{3} \mathrm{He}\left({ }^{3} \mathrm{He}, 2 p\right){ }^{4} \mathrm{He}$ reaction, and thus its rate has a direct effect on our understanding of stellar burning and solar neutrino flux observations. The LUNA Collaboration measured the crosssection down to $16.5 \mathrm{keV}$, where the $S$-factor was observed to increase because of the effect of electron screening [26, 27]. Electron screening effectively reduces the height of the Coulomb barrier in the reaction resulting in an increase in the measured $S$-factor and is typically parameterized in terms of an electron screening potential, $U_{e}$. It was found in ref. [26] that the screening potential needed to reproduce the data was roughly twice the adiabatic (i.e., upper) limit. While this discrepancy is not understood, it is clear that we must understand electron screening because the screening in the stellar plasma is quite different from that in laboratory studies. The cross-sections measured in the laboratory can only be related to the stellar reaction rate if the screening potential is understood. The observed discrepancy may, in part, be due to a broad ${ }^{6} \mathrm{Be}$ resonance near the ${ }^{3} \mathrm{He}+{ }^{3} \mathrm{He}$ threshold at $11.49 \mathrm{MeV}$. Understanding the level structure and determining the properties of missing ${ }^{6}$ Be levels is thus important for understanding the low energy behavior of the ${ }^{3} \mathrm{He}\left({ }^{3} \mathrm{He}, 2 p\right){ }^{4} \mathrm{He}$ reaction and the electron screening potential.

There is considerable evidence that unobserved levels do exist in this excitation energy region of ${ }^{6}$ Be. First of all, several levels have been observed in the isospin mirror nucleus, ${ }^{6} \mathrm{He}$, for which analogs have not been observed in ${ }^{6} \mathrm{Be}[28]$. In particular, two ${ }^{6} \mathrm{He}$ levels at 14.6 and $15.5 \mathrm{MeV}$ should have analog levels in ${ }^{6} \mathrm{Be}$ near or above the ${ }^{3} \mathrm{He}+$ ${ }^{3} \mathrm{He}$ threshold at $11.49 \mathrm{MeV}$. Levels have been predicted at $12.8,14.7$, and $23.8 \mathrm{MeV}$ [29]. Furthermore, a recent measurement at Notre Dame found tentative evidence for a ${ }^{6}$ Be level at $9.6 \mathrm{MeV}$ [30].

To search for these unobserved resonances, we have studied the ${ }^{2} \mathrm{H}\left({ }^{7} \mathrm{Be}, t\right){ }^{6} \mathrm{Be}$ reaction by bombarding a $\sim 1 \mathrm{mg} / \mathrm{cm}^{2} \mathrm{CD}_{2}$ target with a $100 \mathrm{MeV}{ }^{7} \mathrm{Be}^{4+}$ beam. Typical beam intensities were $3 \times 10^{6}{ }^{7} \mathrm{Be} / \mathrm{s}$, and since the beam was fully stripped at the terminal, there was no ${ }^{7} \mathrm{Li}$ contamination in the beam. Tritons were detected and identified by the SIDAR array configured in telescope mode with $100 \mu \mathrm{m}$ thick detectors backed by $500 \mu \mathrm{m}$ thick detectors covering laboratory angles between $\sim 11^{\circ}$ and $33^{\circ}$. With this range of laboratory angles and detector thicknesses, we should be sensitive to ${ }^{6}$ Be levels between $E_{x} \sim 2$ and $13 \mathrm{MeV}$. A typical particle identification plot is shown in fig. 9. Unfortunately, it was found that a large

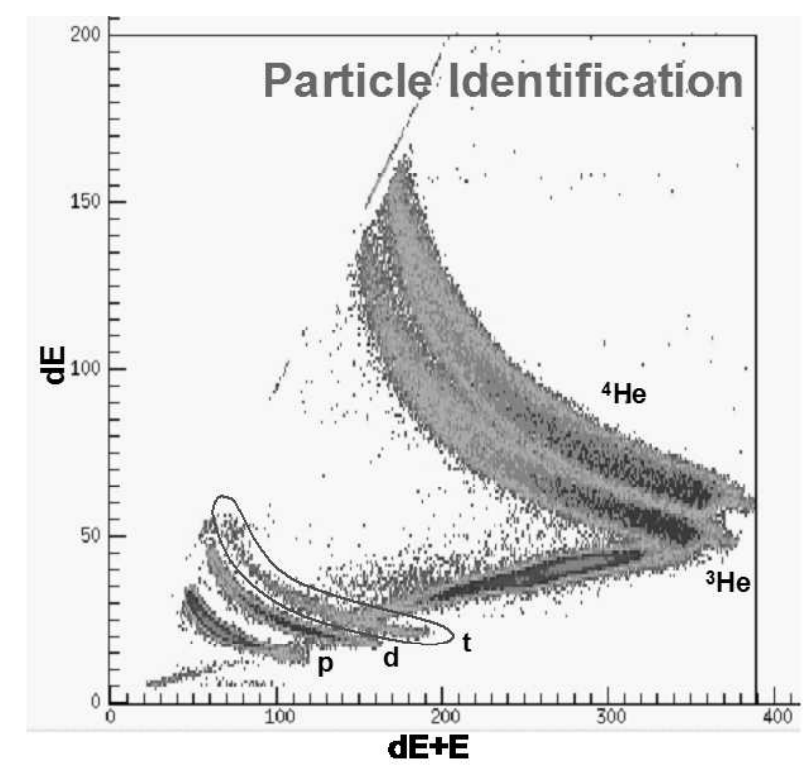

Fig. 9. A particle identification plot from the study of the ${ }^{2} \mathrm{H}\left({ }^{7} \mathrm{Be}, t\right){ }^{6} \mathrm{Be}$ reaction is shown. A veto condition has been applied which reduces the effect of "punch-through" ${ }^{3} \mathrm{He}$ ions near the triton locus by $\sim 95 \%$.

number of high-energy ${ }^{3} \mathrm{He}$ ions are emitted, presumably from $\alpha$ transfer on to carbon in the target. These ${ }^{3} \mathrm{He}$ ions penetrate the stopping detector producing a backbending locus in the particle-identification plot which interferes with the clean identification of tritons. To reduce this effect, a third layer (300 $\mu \mathrm{m}$ thick) of silicon-strip detectors was added to be used as veto detectors. To produce the plot in fig. 9, the absence of a veto detector hit is required. This condition was effective in reducing the punchthrough ${ }^{3} \mathrm{He}$ ions around the triton group by about $95 \%$. Nonetheless, some contamination of the triton group was still observed. Runs were also taken with similar thickness $\mathrm{CH}_{2}$ targets from which it was observed that the number of tritons produced per beam particle was reduced by a factor of 2.4. Therefore, over $70 \%$ of the observed tritons are indeed due to ${ }^{7} \mathrm{Be}+\mathrm{d}$ reactions. This data is currently in a preliminary stage of analysis so no conclusions have yet been drawn concerning the level structure of ${ }^{6} \mathrm{Be}$.

\section{Studies with neutron-rich beams}

The $r$ process produces roughly half of the elements heavier than iron via a series of neutron captures and $\beta$ decays flowing through extremely neutron-rich nuclei. The reaction path and abundances produced are uncertain, however, because of the lack of information on the properties and structure of extremely neutron-rich nuclei. Recently, accelerated beams of many neutron-rich nuclei have become available at the HRIBF [3] making possible some of the first studies of reactions on nuclei along the $r$-process path. While no single measurement is likely to make a tremendous difference in $r$-process calculations, understanding the evolution of shell structure certainly will. 


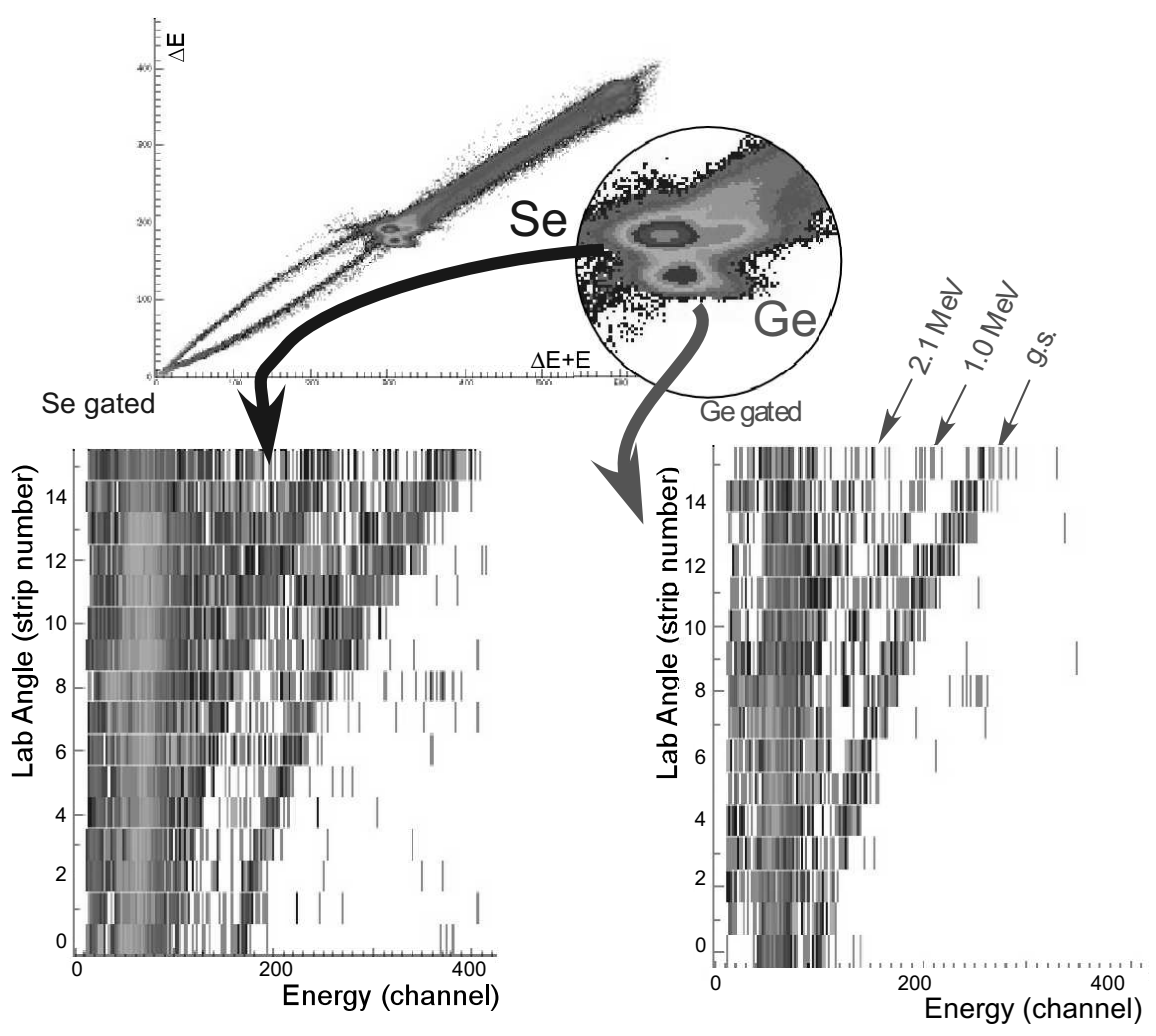

Fig. 10. The top plot shows clear separation of Ge from Se in the ionization counter. By requiring a coincidence with recoil Ge ions, protons detected by the SIDAR array from the ${ }^{2} \mathrm{H}\left({ }^{82} \mathrm{Ge}, p\right){ }^{83} \mathrm{Ge}$ reaction are identified.

The low-lying single-particle structure of neutron-rich nuclei near closed shells is especially important for understanding how the synthesis of elements in the $r$ process may be modified by neutron-capture reactions following the fallout from nuclear statistical equilibrium [31]. The distribution of nuclei concentrates in tightly-bound isotopes near closed shells. As the material cools, neutron captures and $\beta$ decays of these near-closed-shell nuclei alter the abundance pattern. Neutron capture reactions on neutron-rich, closed-shell nuclei are expected to be dominated by direct capture to bound states because of the small $Q$ values for neutron capture and the low-level density in the compound nucleus. Direct capture rates on these nuclei depend sensitively on the structure of lowenergy states - such as energy levels, spins, parities, electromagnetic transition probabilities, and single-particle spectroscopic factors - and typically cannot be accurately estimated in the absence of experimental data [32]. It is, therefore, critical that direct capture rate calculations be supplemented with experimental data near closed shells where the $r$-process abundances peak.

Low-lying single-neutron excitations in ${ }^{83} \mathrm{Ge}$ have been studied for the first time using the ${ }^{2} \mathrm{H}\left({ }^{82} \mathrm{Ge}, p\right){ }^{83} \mathrm{Ge}$ reaction [33]. Previously, only the half-life $\left(t_{1 / 2}=1.85 \mathrm{~s}\right)$ of the $N=51$ nucleus had been measured. The $A=83$ isotope of Ge has seven neutrons more than the last stable Ge nucleus, but it is only one neutron past the $N=50$ closed shell. It is far enough from stability to lie on the path in some $r$-process models, but its low-lying spectrum is still expected to exhibit the simple characteristics of singleparticle structure. With the $(d, p)$ transfer reaction, neutron single-particle states are selectively populated, and proton angular distributions reveal orbital angular momenta and single-particle strengths of final states. Because the mass of ${ }^{82} \mathrm{Ge}$ has been measured, a measurement of the $Q$ value of the reaction also determines the mass of ${ }^{83} \mathrm{Ge}$.

A beam of ${ }^{82} \mathrm{Ge}\left(t_{1 / 2}=4.6 \mathrm{~s}\right)$ was produced at the HRIBF by bombarding a UC target with a $42 \mathrm{MeV}$ proton beam inducing fission of the uranium. Produced $A=82$ ions (Se:Ge:As = 85:15:<1) were accelerated to $330 \mathrm{MeV}$ before delivery to the experiment. Beam currents of ${ }^{82} \mathrm{Ge}$ averaged $10^{4} / \mathrm{s}$. The isobarically mixed $A=82$ beam was used to bombard a $430 \mu \mathrm{g} / \mathrm{cm}^{2} \mathrm{CD}_{2}$ target. Protons from the $(d, p)$ reaction were detected in the SIDAR array at backward laboratory angles $\left(\theta_{l a b}=105^{\circ}-150^{\circ}\right)$ corresponding to forward angles in the center of mass $\left(\theta_{\text {c.m. }}=\right.$ $\left.36^{\circ}-11^{\circ}\right)$. In coincidence with protons, beam-like recoils were detected and identified at forward angles $\left(\theta_{l a b}<1^{\circ}\right)$ in the gas filled ionization counter shown in fig. 1 . The coincidence requirement was necessary to distinguish $(d, p)$ protons induced by ${ }^{82} \mathrm{Ge}$ projectiles from those induced by ${ }^{82} \mathrm{Se}$. This selection is shown in fig. 10. Events from ${ }^{2} \mathrm{H}\left({ }^{82} \mathrm{Ge}, p\right){ }^{83} \mathrm{Ge}$ and ${ }^{2} \mathrm{H}\left({ }^{82} \mathrm{Se}, p\right){ }^{83} \mathrm{Se}$ are clearly visible after the recoil selection.

The $Q$-value for populating the ground state of ${ }^{83} \mathrm{Ge}$ was found to be $1.47 \pm 0.02 \mathrm{MeV}$ resulting in a mass excess of $\Delta\left({ }^{83} \mathrm{Ge}\right)=61.25 \pm 0.26 \mathrm{MeV}$, where the uncertainty is mostly the result of the uncertainty in the mass 


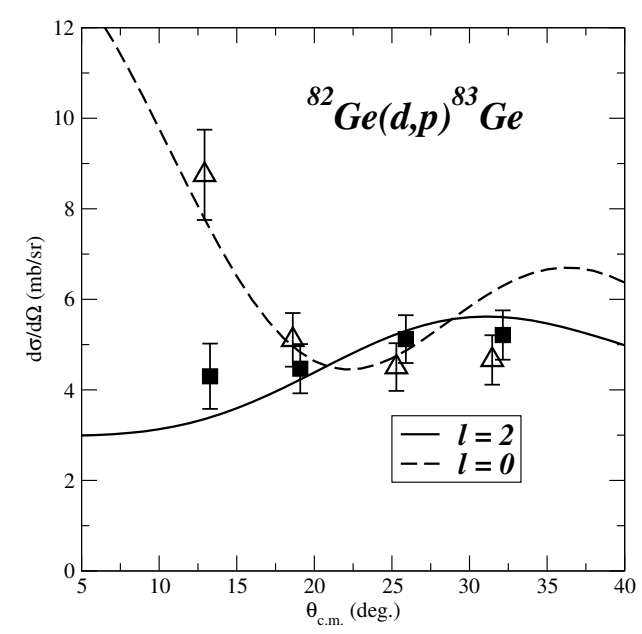

Fig. 11. Proton angular distributions as a function of c.m. angle for ${ }^{83} \mathrm{Ge}$. Ground-state data (filled squares) fitted by $\ell=$ 2 (solid curve); $E_{x}=280 \mathrm{keV}$ data (open triangles) fitted by $\ell=0$ (dashed curve).

of ${ }^{82} \mathrm{Ge}( \pm 244 \mathrm{keV})$. The first excited state was found to be at $E_{x}=280 \pm 20 \mathrm{keV}$. Angular distributions have been extracted for the ground and first excited state and are plotted in fig. 11 .

Distorted-waves Born (DWBA) calculations have been performed to fit the angular distributions in fig. 11. In the present inverse kinematics study, with the silicon array covering backwards laboratory angles, elastic scattering could not be measured. Even so, the global optical model parameters as deduced by Lohr and Haeberli [34] (deuterons) and Varner et al. [35] (protons) are well suited for this mass and energy region. Using the DWBA code TWOFNR [36] and global parameter sets for the optical model potentials from refs. [34,35], the fits in fig. 11 were produced. The best fits supported a $J^{\pi}=5 / 2^{+}$assignment for the ground state and $1 / 2^{+}$assignment for the first excited state with spectroscopic factors of $0.48 \pm 0.14$ and $0.50 \pm 0.15$, respectively.

Our second measurement with neutron-rich beams was a study of the ${ }^{2} \mathrm{H}\left({ }^{84} \mathrm{Se}, p\right){ }^{85}$ Se reaction [37]. Since the reaction kinematics are nearly identical to the ${ }^{82} \mathrm{Ge}$ case, the detector setup was nearly the same as well. A $4.5 \mathrm{MeV} / \mathrm{u}$ $A=84$ beam was used to bombard a $200 \mu \mathrm{g} / \mathrm{cm}^{2} \mathrm{CD}_{2}$ target for nearly 10 days. The higher beam energy and thinner target used in this study resulted in a better energy resolution than in the ${ }^{82} \mathrm{Ge}$ study $\left(\Delta E_{c . m} . \sim 220 \mathrm{keV}\right.$ compared with $\sim 300 \mathrm{keV}$ in the ${ }^{82} \mathrm{Ge}$ case). The beam consisted mainly of ${ }^{84} \mathrm{Br}(93 \%)$ and ${ }^{84} \mathrm{Se}(7 \%)$, and the total beam current was $\sim 9 \times 10^{4}$ pps. ${ }^{85}$ Se levels at $E_{x}=$ $0,0.462,1.114$, and $\sim 1.44 \mathrm{MeV}$ were observed. DWBA fits to the ground and first excited states were again consistent with $5 / 2^{+}$and $1 / 2^{+}$spin/parity assignments with spectroscopic factors of $0.33 \pm 0.08$ and $0.30 \pm 0.08$, respectively. A summary of our results of $(d, p)$ measurements on $N=50$ nuclei is shown in fig. 12 .

We are poised to extend these measurements to the next closed neutron shell at $N=82$ by studying $(d, p)$ reactions on ${ }^{130,132} \mathrm{Sn}$. Transfer reactions using a beam

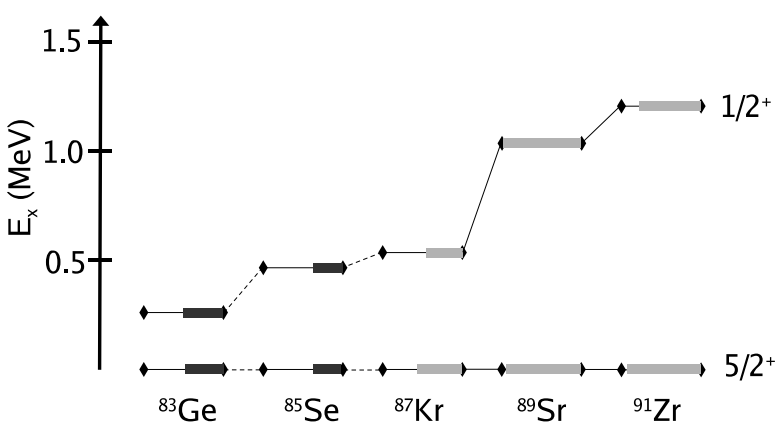

Fig. 12. Spectroscopic properties of the first two states of the even $Z<40, N=51$ isotones. The lengths of the thick lines represent measured spectroscopic factors. Data for ${ }^{83} \mathrm{Ge}$ and ${ }^{85} \mathrm{Se}$ are from the present work. Data for the other $N=51$ isotones: ${ }^{91} \mathrm{Zr}$ [38], ${ }^{89} \mathrm{Sr}$ [39], ${ }^{87} \mathrm{Kr}$ [40].

of ${ }^{132} \mathrm{Sn}$ have been the subject of recent attention and are often singled out as prototypical experiments [41,42, 43] for next generation facilities such as the Rare Isotope Accelerator [44]. At the HRIBF, it is possible to accelerate beams of ${ }^{130,132} \mathrm{Sn}$ to energies around the Coulomb barrier with sufficient intensity to begin such studies.

The $(d, p)$ cross-section to populate low lying, low angular momentum states is often greater at lower beam energies, due to kinematic matching conditions, and at the same time the reaction is cleaner as there are few reaction channels open. However, angular distributions below the Coulomb barrier become less distinctive, and it was not clear that $\ell$ values and spectroscopic factors could be extracted under such conditions. To examine these possible difficulties, we have performed a study of the ${ }^{2} \mathrm{H}\left({ }^{124} \mathrm{Sn}, p\right){ }^{125} \mathrm{Sn}$ reaction [45]. This reaction has been studied at several energies in normal kinematics and was therefore a good benchmark [46,47].

A beam of ${ }^{124} \mathrm{Sn}$ was accelerated to $562 \mathrm{MeV}$ and focused onto a $100 \mu \mathrm{g} / \mathrm{cm}^{2}$ target of deuterated polyethylene $\left(\mathrm{CD}_{2}\right)$. The target was turned $30^{\circ}$ with respect to the beam, thus achieving an effective thickness of $200 \mu \mathrm{g} / \mathrm{cm}^{2}$ and at the same time allowing emerging protons to be detected around $\theta_{l a b}=90^{\circ}$ without being shadowed by the target frame. Protons were detected in two telescopes of silicon detectors. One telescope on the downstream side subtended $\theta_{l a b}=70^{\circ}-102^{\circ}$. The other telescope on the upstream side subtended $\theta_{l a b}=85^{\circ}-110^{\circ}$. The SIDAR array was mounted at more backward angles $\theta_{l a b}=130^{\circ}-160^{\circ}$. The telescopes consisted of a thin position-sensitive $(\Delta E)$ detector and a thicker stopping $(E)$ detector $(1000 \mu \mathrm{m})$. The $\Delta E$ detectors were either 65 or $140 \mu \mathrm{m}$ thick depending on the angles (and thus the proton energies) needed. Both $\Delta E$ detectors had 16 position-sensitive strips allowing the angle of proton emission to be determined to a precision of $\pm 0.5^{\circ}$.

Data were collected for about 18 hours with a beam rate of $10^{7}{ }^{124} \mathrm{Sn} / \mathrm{s}$. The angles of particles measured in the downstream telescope are shown as a function of their energy in fig. 13. There are three clearly visible loci relating to elastically-scattered carbon atoms, deuterons, and 


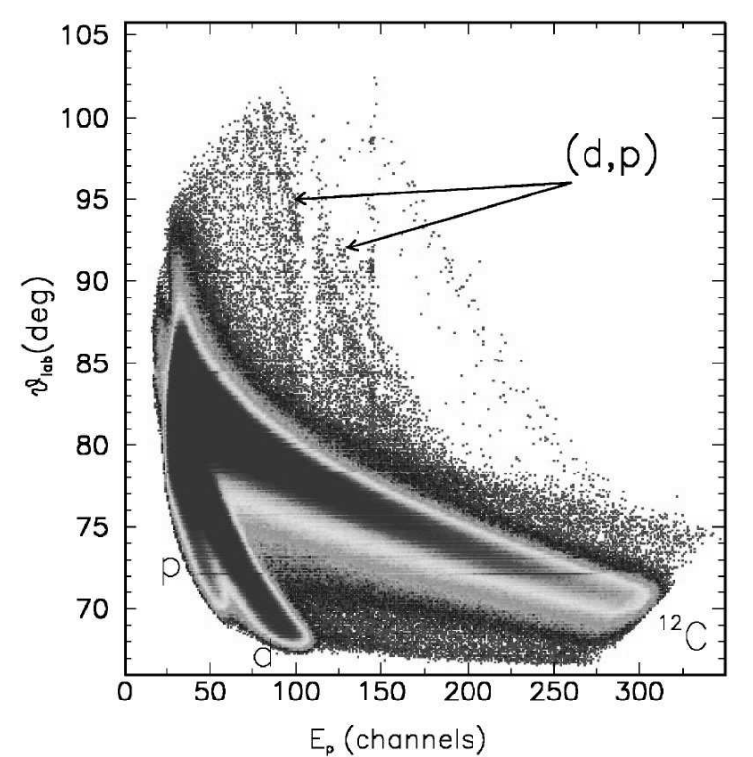

Fig. 13. Angle of particles measured in the ${ }^{2} \mathrm{H}\left({ }^{124} \mathrm{Sn}, p\right){ }^{125} \mathrm{Sn}$ study as a function of energy without any gates imposed. Elastic scattering of carbon atoms, deuterons, and protons from the target and reaction protons from the $(d, p)$ reaction are indicated.

Table 1. Spectroscopic factors from this work and previous works for ${ }^{124} \mathrm{Sn}(d, p){ }^{125} \mathrm{Sn}$. The quoted uncertainties include statistical, DWBA fitting effects, and systematic errors due to the normalization.

\begin{tabular}{ccccc}
\hline$E_{x}(\mathrm{MeV})$ & $J^{\pi}$ & This work & Ref. [47] & Ref. [46] \\
\hline 0.028 & $3 / 2^{+}$ & $0.44(6)$ & 0.53 & 0.44 \\
0.215 & $1 / 2^{+}$ & $0.33(4)$ & 0.32 & 0.33 \\
2.8 & $7 / 2^{-}$ & $0.46(5)$ & 0.52 & 0.54 \\
\hline
\end{tabular}

protons from the target; additionally the distinctive loci resulting from the $(d, p)$ reaction are discernible at larger angles. A break can be seen around channel 100 where protons following the $(d, p)$ reaction have just enough energy to punch through the $\Delta E$ detector, leaving only a subthreshold signal in the $E$ detector. Once an energyloss gate is imposed on the protons, only protons from the $(d, p)$ reaction remain in this plot [45].

${ }^{125} \mathrm{Sn}$ states at $E_{x}=0.028,0.215$, and $2.8 \mathrm{MeV}$ were strongly populated in the study. The resolution in excitation energy was found to be approximately $200 \mathrm{keV}$, owing mainly to target thickness and beam spot size effects. It should be noted that the level density of low-lying states around ${ }^{132} \mathrm{Sn}$ is expected to be low, and thus such energy resolution should be more than sufficient for studies in this nuclide region. Angular distributions have been extracted for these levels [45] with the absolute normalization of the cross-sections determined from the observed angular distribution of elastically-scattered deuterons. The angular distributions from these states show distinctive structures which are characteristic of the angular momentum transferred in the reaction [45]. Spectroscopic factors have been extracted by comparing the measured angular distribu-

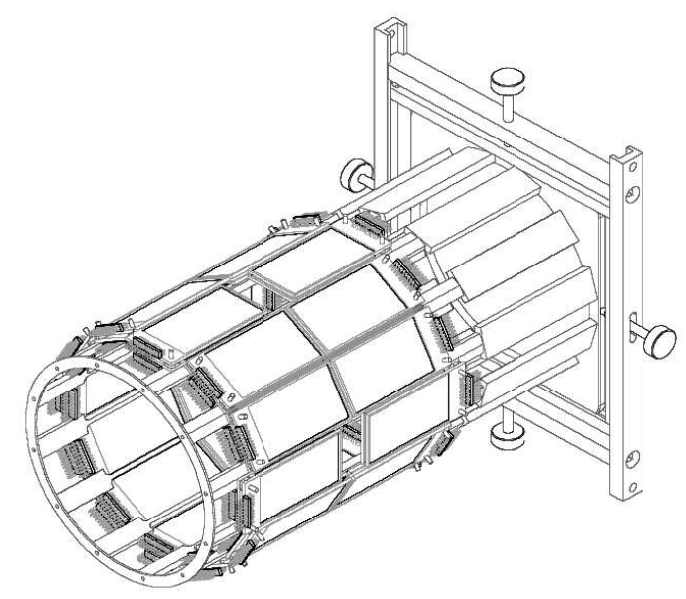

Fig. 14. The ORRUBA detector array showing the two rings of charged-particle telescopes. The gap between rings allows for insertion of the target.

tions with calculations using TWOFNR. The results are shown in table 1 . The values of the spectroscopic factors all agree well with those from the work of refs. $[46,47]$ which were performed in normal kinematics. These results demonstrate the effectiveness of these techniques to determine spectroscopic properties of nuclei in this mass region at beam energies close to the Coulomb barrier.

After the encouraging results obtained in this demonstration measurement, we are developing a larger silicondetector array specifically for the better detection and identification of charged particles emitted near $90^{\circ}$ in the laboratory. The Oak Ridge Rutgers University Barrel Array (ORRUBA) [48] will be a large solid angle silicon detector array, capable of providing energy, angle, and particle identification information. ORRUBA will be comprised of two rings of 12 position-sensitive silicon detector telescopes, symmetrically covering angles forward and backward of $90^{\circ}$ (see fig. 14). Each detector is $\sim 8 \mathrm{~cm}$ long, and its width is divided into four $1 \mathrm{~cm}$ wide resistive strips, oriented parallel to the beam axis. Readouts from both ends of each strip allow measurement of the position of the interaction, allowing determination of the emission angle of the detected particles. Prototype detectors have arrived and are currently being tested.

\section{Conclusions}

Twenty years after Willy Fowler's historic call for data on the properties and interactions of radioactive nuclei [49], scientists have obtained a first glimpse of the possibilities that radioactive beams offer for nuclear astrophysics studies. The HRIBF is one of a few first-generation radioactive beam facilities that has provided some of these first glimpses. We have used accelerated beams of ${ }^{17,18} \mathrm{~F}$ to better understand key reactions that power the nova outburst and produce observational tracers of the explosion such as ${ }^{18} \mathrm{~F}$. Our studies indicate that ${ }^{18} \mathrm{~F}$ is destroyed in the novae environment by the ${ }^{18} \mathrm{~F}(p, \alpha){ }^{15} \mathrm{O}$ reaction at 
a lower rate than previously thought. We have used the tools available at nucastrodata.org to quantify the effect and calculate that roughly a factor of 2 more ${ }^{18} \mathrm{~F}$ is ejected than previously thought.

We are using ${ }^{7}$ Be beams to study reactions of importance to stellar burning. By bombarding a windowless hydrogen target with the ${ }^{7} \mathrm{Be}$ beam and detecting recoil ${ }^{8} \mathrm{~B}$ nuclei at the focal plane of the Daresbury Recoil Separator, we are on the verge of making the first statisticallysignificant measurement of ${ }^{7} \mathrm{Be}(p, \gamma)^{8} \mathrm{~B}$ in inverse kinematics. Only after several measurements with different systematic uncertainties, can the community hope to obtain a truly precise measure of the ${ }^{7} \mathrm{Be}(p, \gamma)^{8} \mathrm{~B}$ reaction rate at stellar energies. We are also studying the unbound nucleus, ${ }^{6} \mathrm{Be}$, via a measurement of the ${ }^{2} \mathrm{H}\left({ }^{7} \mathrm{Be}, t\right){ }^{6} \mathrm{Be}$ reaction at $100 \mathrm{MeV}$. Levels in ${ }^{6} \mathrm{Be}$ are important for understanding the ${ }^{3} \mathrm{He}\left({ }^{3} \mathrm{He}, 2 p\right){ }^{4} \mathrm{He}$ reaction and electron screening at stellar energies.

Finally, we are using radioactive beams of fission fragments to study neutron-rich nuclei near closed neutron closed shells. In particular, we are using the $(d, p)$ reaction in inverse kinematics to probe single-particle excitations. We have made the first neutron-transfer measurement on an $r$ process nucleus, ${ }^{82} \mathrm{Ge}$, and are poised to continue our studies near doubly-magic ${ }^{132} \mathrm{Sn}$. A new detector array, ORRUBA, is being developed to facilitate these studies.

Most of the experiments mentioned here were performed by the Radioactive Ion Beams for Explosive Nucleosynthesis Studies (RIBENS) Collaboration, which is a collaboration of over 30 scientists from 11 institutions including ORNL, Rutgers University, Tennessee Technological University, Colorado School of Mines, Ohio University, Yale University, and the University of North Carolina at Chapel Hill. This research was sponsored, in part, by the Laboratory Directed Research and Development Program of Oak Ridge National Laboratory, managed by UT-Battelle, LLC, for the U.S. Department of Energy under Contract No. DE-AC05-00OR22725.

\section{References}

1. M.S. Smith, K.E. Rehm, Annu. Rev. Nucl. Part. Sci. 51, 91 (2000).

2. G.D. Alton, J.R. Beene, J. Phys. G 24, 1347 (1998).

3. D.W. Stracener, G.D. Alton, R.L. Auble, J.R. Beene, P.E. Mueller, J.C. Bilheux, Nucl. Instrum. Methods Phys. Res. A 521, 126 (2004) and references therein.

4. D.W. Bardayan et al., Phys. Rev. Lett. 83, 45 (1999).

5. C.J. Gross et al., Nucl. Instrum. Methods Phys. Res. A 450, $12(2000)$.

6. M. Hernanz, J. José, A. Coc, J. Gómez-Gomar, J. Isern, Astrophys. J. Lett. 526, L97 (1999).

7. A. Coc, M. Hernanz, J. José, J.-P. Thibaud, Astron. Astrophys. 357, 561 (2000).

8. D.W. Bardayan et al., Phys. Rev. C 62, 042802(R) (2000).

9. R. Coszach et al., Phys. Lett. B 353, 184 (1995).

10. K.E. Rehm et al., Phys. Rev. C 52, R460 (1995); 53, 1950 (1996).
11. D.W. Bardayan et al., Phys. Rev. C 70, 015804 (2004).

12. A. Galindo-Uribarri et al., Nucl. Instrum. Methods Phys. Res. B 172, 647 (2000).

13. N.-C. Shu, D.W. Bardayan, J.C. Blackmon, Y.-S. Chen, R.L. Kozub, P.D. Parker, M.S. Smith, Chin. Phys. Lett. 20, 1470 (2003).

14. D.W. Bardayan et al., Phys. Rev. C 63, 065802 (2001).

15. D.W. Bardayan et al., Phys. Rev. Lett. 89, 262501 (2002).

16. M. Wiescher, K.-U. Kettner, Astrophys. J. 263, 891 (1982).

17. R.L. Kozub et al., Phys. Rev. C 71, 032801(R) (2005).

18. R. Fitzgerald et al., Proceedings of the 6th International Conference on Radioactive Nuclear Beams, Nucl. Phys. A 748, 351 (2005).

19. N. de Séréville et al., Phys. Rev. C 67, 052801(R) (2003).

20. Y. Butt et al., Phys. Rev. C 58, 10(R) (1998).

21. D.W. Bardayan et al., Phys. Rev. C 71, 018801 (2005).

22. S. Parete-Koon et al., Astrophys. J. 598, 1239 (2003).

23. http://www . nucastrodata.org.

24. R. Fitzgerald, Ph. D. Thesis, University of North Carolina at Chapel Hill (2005).

25. R.H. Cyburt, B. Davids, B.K. Jennings, Phys. Rev. C 70, 045801 (2004).

26. M. Junker et al., Phys. Rev. C 57, 2700 (1998).

27. R. Bonetti et al., Phys. Rev. Lett. 82, 5205 (1999).

28. D.R. Tilley et al., Nucl. Phys. A 708, 1 (2002).

29. J.J. Bevelacqua, Phys. Rev. C 33, 699 (1986).

30. V. Guimaraes et al., Nucl. Phys. A 722, 341c (2003).

31. R. Surman, J. Engel, Phys. Rev. C 64, 035801 (2001).

32. T. Rauscher et al., Phys. Rev. C 57, 2031 (1998).

33. J.S. Thomas et al., Phys. Rev. C 71, 021302(R) (2005).

34. J.M. Lohr, W. Haeberli, Nucl. Phys. A 232, 381 (1974).

35. R.L. Varner, W.J. Thompson, T.L. McAbee, E.J. Ludwig, T.B. Clegg, Phys. Rep. 201, 57 (1991).

36. University of Surrey modified version of the code TWOFNR of M. Igarashi, M. Toyama, N. Kishida (private communication).

37. J.S. Thomas, Ph. D. Thesis, Rutgers University (2005).

38. R.D. Rathmell, P.J. Bjorkholm, W. Haeberli, Nucl. Phys. A 206, 459 (1973).

39. T.P. Cleary, Nucl. Phys. A 301, 317 (1978).

40. K. Haravu, C.L. Hollas, P.J. Riley, W.R. Coker, Phys. Rev. C 1, 938 (1970).

41. K.E. Rehm, in Proceedings of the Second International Conference on Fission and Properties of Neutron-Rich Nuclei, St. Andrews, Scotland, 1999, edited by J.H. Hamilton, W.R. Phillips, H.K. Carter (World Scientific, Singapore, 2000) p. 439.

42. ISAC-II A Project for Higher Energies at ISAC (1999), http://www .triumf .ca/ISAC-II/TRI-99-1.pdf.

43. A.H. Wuosmaa, T. Al Tahtamouni, J.P. Schiffer, Nucl. Phys. A 746, 267c (2004).

44. http://www .orau.org/ria.

45. K.L. Jones et al., Phys. Rev. C 70, 067602 (2004).

46. C.R. Bingham, D.L. Hillis, Phys. Rev. C 8, 729 (1973).

47. A. Stromich, B. Steinmetz, R. Bangert, B. Gonsior, M. Roth, P. von Brentano, Phys. Rev. C 16, 2193 (1977).

48. http://www. orau.org/stewardship/.

49. W.A. Fowler, in Proceedings of the Accelerated Radioactive Beams Workshop, edited by L. Buchmann, J.M. D'Auria (TRIUMF Report TRI-85-1, Vancouver, 1985) p. 1. 